\title{
Clinical Effect of Fuming-Washing with Traditional Chinese Medicine after Anorectal Surgery
}

\author{
Shubin Zhu1, Qunhu Feng2* \\ ${ }^{1}$ Shaanxi University of Traditional Chinese Medicine, Xianyang 712000, China \\ 2Shenzhen Bao'an Hospital of Traditional Chinese Medicine, Shenzhen 518000, Guangdong Province, China \\ *Corresponding author: Qunhu Feng, dcfqh2004@aliyun.com
}

\begin{abstract}
Objective: To observe the effect of fuming-washing with traditional Chinese medicine (TCM) after anorectal surgery. Methods: 116 cases of patients with surgical operation were selected in the anorectal department of our hospital, which is Shenzhen Bao'an Hospital of Traditional Chinese Medicine, Shenzhen. According to the different postoperative adjuvant treatment methods implemented by patients, they were divided into two groups under the premise of equal number of patients. Among them, 58 cases of patients with traditional Chinese medicine (TCM) fuming-washing treatment were divided into study group, and patients with hip bath treatment with potassium permanganate solution were divided into common group. The efficacy of adjuvant therapy was observed. Results: The observation and analysis showed that the actual effect of TCM adjuvant therapy was more prominent, and the difference between groups was more significant $(P<0.05)$. Conclusion: The effect of traditional Chinese medicine adjuvant therapy is more significant than that of chemical method, which can help patients achieve better postoperative recovery effect. We should popularize this treatment in time for patients after surgery, and improve the prognosis of patients.
\end{abstract}

Keywords: Anorectal surgery; Surgical treatment; Traditional Chinese medicine fuming-washing; Curative effect

Publication date: July 2021; Online publication: July 31, 2021

\section{Introduction}

In the treatment of anorectal surgical diseases, in order to completely eradicate the focus, surgery is generally used. In the process of applying this method, due to the special regional distribution of the affected area and the characteristics of skin wrinkles, the surgical treatment can only be performed through open incision. In this case, it will bring relatively large psychological pressure to the prognosis of patients. It is necessary to take timely auxiliary treatment measures to promote the healing of the surgical incision, thus to achieve the timely recovery of the surgical wound and achieve the objective. On the other hand, the recovery effect is more significant ${ }^{[1]}$. In this study, 116 patients were selected and divided into groups according to adjuvant therapy. The case selection period was from May 2018 to May 2019, and the detailed overall research process report is described in the following.

\section{Data}

\subsection{General information}

The total number of patients was 116 from May 2018 to May 2019. Combined with different postoperative adjuvant treatment methods, they were divided into study group (herbal fuming-washing) and common group (potassium permanganate hip bath). The general information of patients is as follows: general group, gender distribution, 36 male patients, 22 female patients. Age distribution, the largest 73 years old, the smallest 28 years old, average $(47.03 \pm 3.05)$ years old. The longest course of disease was 6 years and the 
shortest was 3 months. The average age was $(3.92 \pm 1.22)$ years. In the study group, there were 33 male patients and 25 female patients. The age distribution was 73 years old and 27 years old, with an average of $(46.37 \pm 3.28)$ years. The longest course of disease was 6 years and the shortest was 4 months. The average age was $(3.41 \pm 2.01)$ years. There was no statistical difference in the above routine clinical data $(P>0.05)$.

\section{Method}

\subsection{Common group method}

Potassium permanganate was dissolved in sitz bath water for adjuvant treatment. The concentration of potassium permanganate solution was controlled at the level of $0.02 \%$ and dissolved in $1500 \mathrm{ml}$ water. After confirming that the solution was heated to an appropriate temperature, fuming-washing was carried out for adjuvant treatment. The fuming-washing lasted for $10 \mathrm{~min}$. Continuous treatment for 14 days was appropriate, as this kind of treatment belongs to the mode of using chemical drugs for treatment, it is necessary to fully consider the stimulation degree of chemical drugs, and choose this treatment method according to the specific acceptance degree of patients.

\subsection{Research group method}

This set of patients received specific Chinese medicine for fuming-washing. To manufacture fumingwashing basic mixed medicine, basic Chinese medicine such as Radix Sophorae Flavescentis and Fructus were chosen, and a portion of appropriate Chinese medicine was added. The specific prescription selection of TCM can be combined with the pharmacology, therapeutic efficacy and compatibility of different basic drugs. In order to facilitate the use, all drugs are packed in bags according to a certain weight, which can not only ensure the stable efficacy of drugs selected by patients in the process of continuous treatment, but also provide convenience for medical staff to observe and control the process of drug treatment. The volume of liquid medicine in independent package is $10 \mathrm{ml}$.

During the fuming-washingoperation, it is necessary to strictly control the adding order and heating mode of raw materials and basic water, select the gradual heating mode, and reasonably control the temperature index level when it is close to the appropriate temperature, so as to avoid the overheating of water temperature and affect the subjective experience of patients in the fuming-washing process. Before the implementation of fuming-washing, patients should complete defecation to avoid the stimulation of fuming-washing leading to defecation in the treatment process. The total amount of water for fumingwashing should also be maintained within the range of $1500 \mathrm{ml}{ }^{[2]}$. Reasonable water volume and dosage ratio is conducive to obtain the most accurate dosage ratio and promote the best medical effect. Before hip bath, let the medicine reach the surgical wound through steam, and then wait for the water temperature to drop to $40^{\circ} \mathrm{C}$ before hip bath. In the process of adjuvant treatment, the subjective feelings of patients in the process of treatment should be inquired in time. In case of discomfort, the fuming-washing treatment should be stopped in time. The duration of hip bath should be maintained at 15 minutes for 14 consecutive days.

\section{Observation indexes}

Combined with different postoperative adjuvant treatment methods, the specific treatment effect was observed, and the evaluation standard was divided according to the effect significance.

\section{Statistical methods}

In the process of this study, SPSS 20.0 was selected as the professional statistical software, and $P$ was used as the main basis for judging the statistical results. When $P<0.05$, the results were statistically significant. 


\section{Results}

To observe the auxiliary promotion effect of disease treatment, it can be seen that the research group using TCM as the auxiliary treatment drug has higher total effectiveness index level, and the comparison between groups shows significant statistical difference $(P<0.05)$. The detailed statistical results are shown in Table 1. below.

Table 1. Statistical table of the effectiveness of disease adjuvant therapy between groups

\begin{tabular}{cccccc}
\hline Group group & $\begin{array}{c}\text { Number of } \\
\text { patients }\end{array}$ & $\begin{array}{c}\text { Very } \\
\text { effective }\end{array}$ & $\begin{array}{c}\text { Basically } \\
\text { effective }\end{array}$ & $\begin{array}{c}\text { Basically } \\
\text { invalid }\end{array}$ & $\begin{array}{c}\text { Total effective } \\
\text { rate }\end{array}$ \\
\hline General group & 58 & 24 & 21 & 13 & $77.59 \%$ \\
Research Group & 58 & 37 & 19 & 2 & $96.55 \%$ \\
$P$ & & & & & $<0.05$ \\
\hline
\end{tabular}

\section{Discussion}

For patients undergoing anorectal surgery, the recovery stage of surgical wound is a relatively painful stage for patients. If measures are not taken in time for adjuvant treatment and nursing, the overall recovery period will be prolonged and the recovery effect will be negatively affected. Therefore, the choice of appropriate adjuvant treatment measures is an important condition to achieve more effective nursing effect.

In the results of this study, the research group of patients with TCM fuming-washing auxiliary treatment method for better treatment effect has a very significant role in promoting, the overall treatment efficiency level is higher, reaching $96.55 \%$, compared with the ordinary group, the statistical difference is significant $(\mathrm{P}<0.05)$.

To sum up, after the implementation of anorectal surgery, in a variety of different adjuvant treatment measures, herbal fuming-washing is more effective than chemical therapy. After this kind of operation, TCM should be preferred as an adjuvant drug for fuming-washing.

\section{Disclosure statement}

The author declares no conflict of interest.

\section{References}

[1] Zhu YY, Sheng GL, Shen JY, et al., 2019, Observation on the Curative Effect of Single Stapler Hemorrhoidectomy Combined with Blood-activating and Stasis-resolving and Heat-clearing and Dampness-resolving Fuming-washing Therapy in the Treatment of Moderate and Severe Rectocele Complicated with Mixed Hemorrhoids. Chinese Journal of Medicine and Clinic, 19(9): 1501-1503.

[2] Ma LJ, Tan KL, Zhang S, et al., 2019, Meta Analysis of the Effect of Acupuncture Combined with Traditional Chinese Medicine Fuming-Washing and Hip Bath on Postoperative Pain of Anorectal Diseases. Modern Chinese Medicine Research and Practice, 33(5): 62-68. 